\title{
New Media
}

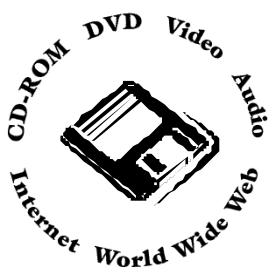

\section{CD-ROM Review: The Evolution of the Anesthesia Machine}

Copyright 2000 by Rafael Ortega MD,

Harold Arkoff MD.

Distributed and sponsored by Datex-Ohmeda.

This Windows CD-ROM is a marvelous historical survey of the evolution of the technology used in our profession. It was produced using Macromedia Director and in collaboration with the Wood Library Museum of the American Society of Anesthesiologists where Dr. Ortega was awarded an anesthesia history fellowship. It was, quite justly, awarded first prize for being the outstanding scientific and educational exhibit at the ASA annual meeting in 1998. Both authors were affiliated with Boston University at the time. It was developed "in an effort to enhance safety in anesthesiology through the understanding of its history."

I had no trouble getting the software to install on my test computer, a $300 \mathrm{MHz}$ Pentium II laptop running Windows 98. The CD-ROM will automatically play when placed in the CD-ROM drive. The setup program will add a menu to your Start Program Menu, as well as add Microsoft DirectX and Microsoft Media Player capability where needed. (Microsoft DirectX and Media Player are required to play the media content of "Evolution of the Anesthesia Machine".) There is a nice option to run the software entirely from CD-ROM, which will appeal to many people with limited disk space.

As the title suggests, this is a CD-ROM about the history of the anesthesia machine. In fact, the focus of the CD-ROM goes beyond mere history of anesthesia technology to additionally offer a considerable amount of material dealing with the American origins of ether anesthesia, starting, of course, with the details of Morton's public demonstration of ether anesthesia at the "Ether Dome" of Massachusetts General Hospital on October 16, 1846. To place this date in the context of contemporary European affairs, there is even a small section covering the key historical events of the time, such as the publication of the Communist Manifesto in 1848. These kinds of little extras help make this CD-ROM a really attractive product.
A particularly nice feature of the CD-ROM is the "timeline" section, which covers the history of anesthesia from the 1840s to the present, one decade at a time. At each decade, a series of icons is offered dealing with such topics as the Allis ether inhaler (1874) or the Richardson ether bottle (1920). Some of these sections have associated video clips. Another interesting feature is a series of brief interviews with historically important individuals such as Dr. Leroy Vandam or recent individuals involved in advancing anesthesia technology.

I found the most delightful aspect of the CD-ROM to be a series of short black and white video clips teaching how to use open drop ether anesthesia using a Shimmelbusch face mask. The videos show real historical footage of a patient receiving ether anesthesia, complete with an episode of laryngospasm.

The CD-ROM closes with a look at anesthesia developments we can expect to see in the future, as exemplified by video clips of interviews with an advanced anesthesia machine designer at Ohmeda and with the inventor of the bi-spectral index (BIS) monitor.

While it is true that this CD-ROM would be of relatively little value in preparing for the anesthesia fellowship examination, it will almost certainly delight those practitioners interested in knowing how our forefathers provided anesthesia care. Think of it as an anesthesia museum on CD-ROM. Highly recommended.

\section{John Doyle MD PhD FRCPC}

Toronto, Ontario

\section{Embedded Computers in the Operating Room and in the Intensive Cave Unit}

Computers are now an important and integral part of modern anesthesia care. In many cases "embedded" computers are integrated "transparently and seamlessly" into medical instruments so that the user never realizes that he or she is using a computer per se. 
The incorporation of embedded computer technology into monitoring devices along with recent developments in clinical data management is now starting to provide clinicians with the potential for improved decision-making capability. Computerization has also simplified the use of systems for the target-controlled and closed-loop delivery of intravenous and inhaled drugs. As these developments continue we can expect that the use of computer technology will steadily increase over the next several years.

Indeed, embedded computers are used in virtually all medical instrumentation used in the operating room and intensive care unit, similar to chips in TVs, VCRs, microwave ovens, etc. Such instruments include blood pressure monitors, pulse oximeters, capnographs, anesthetic agent analyzers, and even patient controlled analgesia machines and electrocautery devices. In particular, embedded computer technology has had a profound effect on the evolution of the modern anesthesia machine. Flow control, alarm management, data logging are all examples of activities carried out by the embedded computers present in the latest generations of anesthesia machine. Similarly, the embedded computers present in the latest generations of ventilators carry out data display, flow control, alarm management, and data logging. Finally, many state-of-the-art anesthesia machines will produce an automatic anesthetic record.

Very often, the computers inside these instruments are based on one or more inexpensive single chip systems programmed in "assembly language" (a lowlevel, tedious programming environment), although some recent designs often involve miniaturized and simplified regular IBM personal computer-type designs, and allow for software development using regular computer languages such as Visual Basic or $\mathrm{C}++$. Such embedded computers often consist of a single chip that can cost as little as a dollar or less. Many instruments contain several embedded computers, each focusing on a particular task.

In general such chips are 8,16 or 32 bit devices with non volatile memory (ROM) containing a control program, as well as read/write memory, analogto-digital conversion capability and so on. What differentiates medical embedded computer systems from other kinds of embedded computers is the need for high reliability, the need to ensure that the software meets extremely high standards, and the need for detailed documentation. The American Food and Drug Administration (http://www.fda.gov) is active in defining requirements for such medical embedded computers. This partly explains the relatively high cost of medical equipment compared with common household consumer items such as TVs and VCRs.

Despite the advantages offered by these developments, there is much to be done to employ embedded computers more wisely. All too often the need for a clear and intuitive user interface is forgotten in the race to bring the latest generation product to market. For instance, the epidural and intravenous patientcontrolled analgesia (PCA) systems used in my hospital have serious ergonomic flaws that make them inappropriately difficult to use. (Example: neither system provides visual feedback to the patient (such as a red/green light) to indicate whether or not the patient is "locked out".) The FDA is also aware of these ergonomic issues and is working to offer guidance regarding the best way to use embedded computers in medical equipment that users are comfortable with.

Similarly, there are a number of inherent problems with current intra-operative monitoring systems based on embedded computer technology. First, these systems are necessarily software based, so that all the problems associated with software (testing for bugs, maintenance, upgrades, documentation, etc.) apply. Secondly, as already alluded to, the complexity of such advanced systems usually requires that careful application of human factors (ergonomic) principles be used to ensure that the system is easy to configure and use. For instance, one must ensure that the alarm systems associated with the system are not so "painful" to use that users disable all alarms to get around a high rate of false alarms. Third, software systems are subject to crashes, sometimes at critical times. With respect to this, special attention should be directed to designing the system to recover quickly from system crashes, should they occur. Of course, special attention should also be directed to preventing system crashes in the first place.

Other "must have" features of systems using embedded computers is that they should allow system components to be added or removed without disturbing operation. As an example, users often turn off airway gas monitoring while patients are on cardiopulmonary bypass - it is thus important that when the airway gas monitor is turned off or back on that this does not crash the system. Another issue is that modern patient monitors are often networked, so that data on one monitor can be viewed remotely, for instance in the anesthesia lounge or in another OR. Some monitors contain sufficiently rich data logging capability that they are easily upgraded to full automatic charting systems. Some monitors will run exter- 
nal software such as drug dosing calculation software or medical diagnostic software to identify acid-base disorders from arterial blood gas data. Other monitors allow seamless transfer of data from bedside monitor to transport monitor, and back to the bedside simply by moving a data module.

The next few years will likely witness a continuing boom in the use of computer technology in the OR, but do not expect that the systems will always be easy to use.

D. John Doyle MD PhD FRCPC

Toronto, Ontario

\section{Review of Airway Cam Video Series Volume 2: Pediatric Intubation}

27 min; \$150 (US); written and produced by Richard M. Levitan, Scott Cook-Sather, 1998.

VHS NTSC format. www.airwaycam.com

This is the second in an excellent series of instructional videos by Richard M. Levitan using the Airway $\mathrm{Cam}^{\mathrm{TM}}$ as means of recording real intubations. The first tape in the series, dealing with intubation in adults, received excellent reviews, so I was curious as to whether or not Levitan would succeed once again. I was not disappointed.

A six-step approach to intubation is presented and repeatedly emphasized to drive home the fundamental teaching message. The main anatomical differences between adult and pediatric airways are explained, along with a discussion concerning the equipment differences that stem from anatomical differences. Rules for selecting laryngoscope type and size, as well as guidelines for selecting endotracheal tube type and size are well covered. As with the first video, the emphasis is on learning by example, and we are offered clip after clip of pediatric airway views along with superb quality (indeed, professional) narration describing what is going on. Freeze frames, arrows, and graphics highlight key landmarks and teaching points.

This excellent teaching resource deserves a place in the video library of every anesthesia department. I eagerly await further videos from Dr. Levitan.

D. John Doyle MD PhD FRCPC

Toronto, Ontario

\section{Erratum}

Gray C, Swinhoe CF, Myint $\Upsilon$, Mason D. Target controlled infusion of ketamine as analgesia for TIVA with propofol. Can J Anesth 1999; 46: 957-61.

An error was made in Table I Pharmacokinetic parameters of ketamine used for computer model, page 958 (October issue). The same values to $k_{21}$ and $\mathrm{k}_{12}$ were erroneously assigned. The correct values should be:

$$
\begin{array}{lll}
\mathrm{k}_{21} & 0.01473 \pm 0.003 & \min ^{-1} \\
\mathrm{~K}_{12} & 0.03296 \pm 0.005 & \min ^{-1}
\end{array}
$$

